\title{
QUIZÁS, QUIZÁS, QUIZÁS: LA SELECCIÓN MODAL PARA EXPRESAR PROBABILIDAD EN ESPAÑOL
}

Lorena Camacho Guardado

Universidad de Texas en Dallas

http://dx.doi.org/10.18778/8220-201-4.04

\section{Resumen}

En el aula de E/LE, una de las funciones comunicativas que más problemas presenta es la expresión de la probabilidad. Y es que para dicha función contamos con numerosos mecanismos tanto de modalidad interna como externa que, además, pueden seleccionar más de un modo. El problema está tanto en la terminología empleada para las formas del sistema verbal como en las descripciones poco operativas que se limitan a clasificar dichos mecanismos según sus esquemas sintácticos de subordinación y qué modo selecciona cada uno de ellos, dejando de lado cualquier valor pragmático de estos.

Palabras clave: Probabilidad, selección modal, aproximación, gramática cognitiva.

\section{1.}

\section{Mecanismos para expresar probabilidad en español}

Para expresar lo que no se sabe o la falta de compromiso con la verdad de lo que se enuncia, en español se dispone de una gran variedad de medios de expresión: verbos cognitivos (creer, pensar, suponer, dudar, parecer...), verbos modales (poder, deber (de), 
tener que, haber de...), sustantivos (tengo la duda de que/si, existe la posibilidad de que...), adjetivos (es posible/probable que...), adverbios (quizá, tal vez, posiblemente, probablemente...), así como las formas del modo aproximativo (también llamado condicionado o potencial). Por tanto, los mecanismos formales para expresar probabilidad son tanto de modalidad externa como interna.

En la modalidad interna, la actitud del hablante queda reflejada mediante recursos gramaticales como el modo y el tiempo verbal; sin embargo, en un enunciado de modalidad externa aparecen unidades léxicas (verbo, adjetivo, sustantivo y adverbio) cuyo significado alude a una actitud psicológica o perspectiva modal del hablante, lo que indica que este es capaz de objetivar su actitud haciéndola explícita (Carbonero Cano, 1997: 67).

\section{1. \\ Dificultades}

A la hora de llevar al aula de E/LE la expresión de la probabilidad, muchas son las dificultades que se pueden encontrar. La primera es que, debido a la simplificación en la interpretación de las denominaciones tradicionales de las formas verbales, se cree que los llamados 'tiempos verbales' expresan únicamente tiempo en su sentido cronológico y este es uno de los mayores problemas que se nos presenta a la hora de introducir los distintos mecanismos de modalidad interna (cantaré, habré cantado, cantaría y habría cantado).

Además, nos encontramos con que algunos de estos mecanismos seleccionan un modo (es posible que...), mientras que otros pueden seleccionar tanto indicativo como aproximativo y/o subjuntivo (quizá, probablemente, posiblemente, seguramente...). Para explicar la selección modal en esta función comunicativa, se suele dar una constante mezcla de criterios semántico, sintáctico y pragmático que resulta en descripciones poco operativas (Ruiz Campillo, 1998: 91): que un morfema no tiene significado alguno (justificación sintáctica), creer que el significado puede describirse independientemente de las restricciones impuestas por la estructura de la información (justificación semántica) 
o seleccionar la perspectiva exclusiva que en cada caso convenga, despreciando la solución unitaria del problema (justificación de la mezcla de criterios).

Asimismo, es necesario tener en cuenta el valor pragmático (no solo semántico) de los distintos mecanismos, pues esto explicaría por qué algunos -como a lo mejor, igual o lo mismo- no pueden seleccionar subjuntivo. En este sentido, Bañón Hernández (1999: 138) señala que a lo mejor no es una muestra de duda, sino que transmite la información como segura, pues funciona como locución-signo que el hablante emite cuando quiere convencer a su interlocutor de la seguridad o realidad de su juicio al connotar su opinión con ésta, transmitiendo así una fuerza probable-segura, hecho que justificaría su uso con indicativo. Por tanto, semánticamente se acercaría más a la certeza que a la duda y suele responder a un intento por ser diplomático o cortés en el acto comunicativo y su función básica es la de atenuante pragmático de la responsabilidad del hablante en relación con la verdad de lo dicho (Briz, 1995: 111). Así pues, a lo mejor, igual o lo mismo no expresan mínima posibilidad como se venía defendiendo en algunas gramáticas de E/LE hasta ahora, sino máxima.

Por otro lado, algunas gramáticas y métodos de E/LE han intentado proponer una escala de probabilidad atendiendo al mayor o menor grado de seguridad que el hablante tiene sobre el hecho que refiere. Con todo, esto no es posible-incluso intentando tener en cuenta una virtualidad semántica y otra pragmática de cada uno de los mecanismos para expresar dicha función comunicativa-, debido tanto a la subjetividad emisiva como a la receptiva, ya que es necesario tener en cuenta tanto la intencionalidad del hablante como la interpretación del interlocutor. Así pues, es necesario diseñar métodos didácticos que muestren de forma gradual los criterios semántico, sintáctico y pragmático para, posteriormente, revelar que estos están conectados. 


\section{Los modos del español}

El sistema verbal y modal posee una gran versatilidad, ya que el verbo puede expresar de diversas formas algunas de sus categorías para transmitir determinados mensajes (Está en clase / Estará en clase). Así, se cancela el valor de la categoría gramatical 'tiempo', generando una interpretación modal del morfema, por lo que se ahorra la creación de un morfema específico para cada expresión o la utilización de una estructura sintáctica de mayor complejidad, produciéndose una doble significación que no es más que un procedimiento del sistema para multiplicar la capacidad expresiva de la lengua con el mismo número de formas (Real Espinosa, 2005: 2-3).

Un argumento semántico a favor de considerar las formas del futuro y condicional no como tiempos, sino como un modo independiente, es que suponen algo no realizado y cuyo enunciado no puede ser declarado verdadero o falso al igual que las formas del indicativo.

Algunos gramáticos han sabido reestructurar el sistema verbal español separando dichas formas muy acertadamente, entre ellos Alarcos (1970), pero fue Matte Bon (1992) quien mejoró la capacidad explicativa de la clasificación modal suponiendo valores más generales y operativos en cada modo. Su aportación esencial fue tener en cuenta el contexto discursivo dentro del que se enmarca una comunicación determinada, en la que los interlocutores se intercambian informaciones o las dan por sabidas. Sin embargo, Ruiz Campillo (1998) señala que el concepto de 'información' como valor asociado a la selección modal "se siente más bien como una de las varias posibles consecuencias interpretativas de algo más básico que realmente pueda ser considerado un acto del hablante capaz de transmitirla" y que él identifica como 'declaración' (poner de relieve un estado de cosas). El modo es una pura representación de actos que el hablante manipula a su conveniencia. Aunque reduce el valor del modo a 'declaración' (indicativo) y 'no-declaración' (subjuntivo), afirma que existen precisiones con respecto al diferente 'modo' en que es posible declarar o evitar una 
declaración. Aun concediendo el valor declarativo a futuro y condicional, reconoce que es evidente el carácter modal del contraste Está en la cárcel/Estará en la cárcel o Dijo que sí/Diría que sí, por lo que defiende la existencia de un modo aproximativo/predictivo que es el que nos parece más adecuado para la enseñanza de español como lengua extranjera.

Con todo, en cuanto a la expresión de la probabilidad, las nociones de declaración/no-declaración no terminan de explicar el hecho de que haya adverbios o locuciones adverbiales que seleccionen los tres modos (probablemente, posiblemente, seguramente...) y otros (a lo mejor, lo mismo, igual) que no puedan seleccionar subjuntivo.

\section{1}

\section{Nuevos valores centrales}

Puesto que los valores centrales propuestos por estos autores no sirven para explicar la selección modal de los distintos mecanismos de modalidad externa para expresar probabilidad en español, debemos seguir buscando unos que nos permitan explicar de forma operativa su funcionamiento.

Si nos detenemos en los siguientes enunciados, podemos ver claramente la diferencia de modalidad existente entre ellos:

(1)
a. Está en casa
b. Estará en casa
c. Es posible que esté en casa

a. Volvió a casa

b. Volvería a casa

c. Puede que volviera a casa

Ya se ha mencionado que las formas de futuro y del condicional expresan algo no realizado y se diferencian de las formas del indicativo porque expresan enunciados que no pueden interpretarse como verdaderos o falsos, ya que lo que tenemos es la opinión o valoración aproximada que de un hecho emite el hablante. Por lo tanto, el interlocutor no debe interpretar el mensaje de 
aquel como algo que se va a producir, sino como la aproximación sobre el hecho mismo.

Podemos comprobar que los ejemplos de (1a) y (2a) no son más que una información positiva sobre el presente y el pasado; los de (1b) y (2b) lo hacen de una forma aproximativa. Es decir, son informaciones sobre lo que contemplamos dentro de nuestra realidad o contexto inmediato: lo que conocemos -porque es un hecho- (indicativo) o lo que desconocemos, pero consideramos posible en él (aproximativo). Sin embargo, el subjuntivo no informa sobre hechos (evidentes o probables), sino que expresa ideas que el hablante no considera como parte de su contexto inmediato (porque las rechaza, las cuestiona o considera mínima su posibilidad).

El siguiente dibujo, basado en los estudios realizados por Langacker (1991) y Castañeda Castro (2004b), nos permite explicar de forma significativa el sistema modal español, dejando de lado cualquier criterio temporal como valor exclusivo de cada una de sus formas:

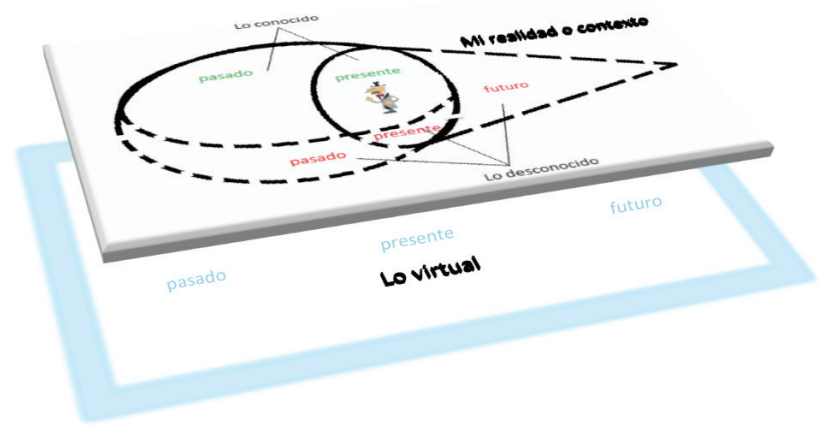

Figura 1. Nuevos valores centrales para los modos del español

El dibujo, a diferencia del propuesto por Castañeda Castro ${ }^{1}$ (2004b: 56), se compone de dos planos o niveles: el de mi mundo

$1 \mathrm{El}$ autor habla de espacios en el mundo representado por el sujeto conceptualizador. 
o contexto inmediato y el plano de lo virtual. En el primero, estaría lo que conozco -porque es un hecho o considero que lo es- y lo que desconozco - pero considero que puede ser un hecho- en pasado, presente o futuro. Las líneas discontinuas hacen referencia a lo desconocido, mientras que las continuas se refieren a lo que conoce el hablante. En el plano virtual, estaría todo aquello que el hablante rechaza, cuestiona, considera mínima su posibilidad o no existe aún para él.

Así pues, el sistema verbal quedaría de la siguiente forma:

Tabla 1. Formas del sistema verbal español

\begin{tabular}{lllll}
\hline Lo conocido & $\begin{array}{l}\text { había } \\
\text { cantado }\end{array}$ & canté/cantaba & he cantado & canto \\
\hline Lo desconocido & $\begin{array}{l}\text { habría } \\
\text { cantado }\end{array}$ & cantaría & $\begin{array}{l}\text { habré } \\
\text { cantado }\end{array}$ & cantaré \\
\hline \multirow{2}{*}{ Lo virtual } & $\begin{array}{l}\text { hubiera/ } \\
\text { hubiese } \\
\text { cantado }\end{array}$ & $\begin{array}{l}\text { cantara/ } \\
\text { cantase }\end{array}$ & haya cantado & cante \\
\hline
\end{tabular}

\section{3.}

\section{El modo en mecanismos de modalidad externa}

La selección modal para expresar probabilidad depende no solo de factores perceptivos o psicológicos, sino de procesos cognitivos del hablante, por lo que urge la necesidad de actualizar el enfoque comunicativo en el ámbito del español como lengua extranjera. Debemos pasar, pues, de una teoría del lenguaje basada en las formas, a una teoría basada en el significado, ya que solo así podremos instruir al aprendiente no sobre cuándo debe usar presente de indicativo, presente de aproximativo o presente de subjuntivo junto a mecanismos de modalidad externa como quizá, probablemente, posiblemente, seguramente..., sino ayudarle a comprender 
dicho significado, para que pueda escoger las formas que mejor representen su pensamiento y que logre autonomía en su proceso de aprendizaje.

\section{1. \\ Secuenciación de mecanismos para expresar probabilidad}

Ante todo, la primera necesidad es la descripción coherente del sistema verbal para poder hacer más fácil el proceso de enseñanza/aprendizaje no ya de la expresión de la probabilidad, sino del español como L2/LE. El futuro y el condicional no pueden ser considerados como tiempos sino como un modo independiente y particular: el aproximativo. Dichas formas deben presentarse por primera vez en el aula en un nivel A2+ pues, si se hace más tardíamente, el aprendiente no las adquirirá con sus valores modales, sino temporales ${ }^{2}$.

En cuanto a los mecanismos de modalidad externa, los significados epistémicos se adquieren tardíamente en una L2/LE, y es que en los niveles elementales el conocimiento de los aprendientes es léxico; es decir, poseen un bajo grado de gramaticalización de su competencia. Así, recurren en primer lugar a emplear los elementos léxicos más transparentes como son los verbos cognitivos (creer, pensar, suponer, parecer...), para pasar después a los adverbios de probabilidad (quizá, tal vez, posiblemente...). Los verbos modales (poder, deber (de), tener que...), en cambio, son los que mayor dificultad de comprensión y adquisición presentan por su ambigüedad e indeterminación.

Teniendo en cuenta esto, el orden debería ser el siguiente:

a) Verbos cognitivos creer, pensar, suponery parecer en nivel A1.

b) Formas aproximativas, junto con los adverbios probablemente, posiblemente y seguramente (con indicativo/aproximativo) en nivel $\mathrm{A} 2+$.

2 Es preferible no presentar cantaré con valor de futuridad, ya que esto puede obstaculizar no ya el aprendizaje, sino la adquisición de su valor real modal. 
c) Mecanismos de modalidad externa que seleccionan estos dos modos en nivel B1. Ampliar los verbos cognitivos (imaginarse, figurarse), presentar otros adverbios como quizá, a lo mejor, igual, lo mismo, tal vez con indicativo o aproximativo, y una primera introducción sobre los verbos modales con valor epistémico (tener que, deber, deber de, poder).

d) Mecanismos de modalidad externa que seleccionan los tres modos en nivel B1+ (para comprobar que no es necesario el uso de subjuntivo). Las formas para expresar probabilidad ya vistas que permiten seleccionar indicativo, aproximativo y/o subjuntivo (seguramente, probablemente, posiblemente, quizá, a lo mejor, igual, lo mismo, tal vez...).

e) Mecanismos de modalidad externa que seleccionan subjuntivo y verlos en contraste con el indicativo en nivel B2. Oraciones subordinadas sustantivas en función de sujeto (es posible que, es probable que, puede (ser) que...), así como mecanismos de probabilidad mediante sustantivos (tengo la duda de que/si, existe la posibilidad de que...).

\section{2.}

\section{Aplicación de estos nuevos valores}

Para comprobar si estos valores son aplicables a los contextos de uso de la expresión de la probabilidad mediante mecanismos de modalidad externa, se propone el siguiente diálogo:

(3)

a. ¡Ayer vi a Bea y Miguel en el cine.

Es un hecho.

b. ¿En serio? ¡A lo mejor son novios!

Lo desconoce, pero lo contempla como probable en su realidad.

c. No os precipitéis; puede que solo sean muy buenos amigos.

Considera mínima la posibilidad de solo ser amigos.

d. Es posible; pero creo que son más que amigos.

Considera muy probable que haya algo entre ellos y mínima la posibilidad de que solo sean amigos. 
e. Tienen que estar saliendo, porque pasan mucho tiempo juntos.

Las circunstancias le obligan a considerar muy probable que estén saliendo.

f. No creo que lo estén; Bea me lo habría dicho.

Cuestiona esa idea, porque Bea no le dijo nada, pero cree que de ser cierto se lo diría.

g. Igual no quiere contarlo.

Lo desconoce, pero lo contempla como probable en su realidad.

h. Quizá no haya nada que contar.

Considera mínima la posibilidad de haber algo que contar.

i. Yo digo que son más que novios.

Es un hecho.

j. Supongo que lo son; el otro día los vimos abrazados.

Desconoce si son novios, pero lo contempla como probable en su realidad, porque los ha visto en el cine.

k. Bea y Miguel son novios; él me lo dijo ayer.

Es un hecho que lo son, porque Miguel se lo ha dicho.

I. ¡No puede ser que estén saliendo; me niego a creer que sean novios!

Rechaza esa idea; por lo tanto, no forma parte de su realidad.

m. ¡Bea y Miguel se casan este verano!

Lo sabe; es un hecho.

n. Quizá es un poco pronto, ¿no?

Lo desconoce, pero lo ve posible.

o. Tal vez lo sea, pero ellos han tomado esa decisión.

Considera mínima la posibilidad de que sea pronto; no la contempla como posible en su realidad, pero es un hecho que han decidido casarse.

p. ¡Lo mismo están embarazados!

Desconoce la causa del casamiento, pero ofrece una de las muchas posibilidades contempladas en su realidad. 
q. Dudo mucho que sea por eso.

Cuestiona esa idea; no la contempla como posible en su realidad.

r. Si estuviera embarazada, me lo habría dicho, ¿no?

Considera mínima la posibilidad del embarazo, porque en ese caso, lo esperado era contárselo.

s. Puede ser que no quiera que la gente lo sepa.

Ofrece una posibilidad que ella misma no considera posible o no quiere comprometerse con lo dicho.

t. ¡Sois unos cotillas! ¡Se casan porque se quieren! Son hechos.

u. Tienes toda la razón.

Es un hecho.

v. ¡No me puedo creer que se vayan a casar!

Esa idea no forma parte de su realidad; no la contempla como posible.

w. Pues ya tienen fecha.

Es un hecho.

\section{4. \\ Conclusiones}

Aunque el aprendiente no emplee en su discurso muchos de los mecanismos formales para expresar probabilidad, necesita conocer el amplio abanico de recursos disponibles para poder interpretar correctamente el mensaje de su interlocutor. Por tanto, urge la necesidad de propuestas adecuadas que ofrezcan una descripción coherente del sistema verbal y modal español para facilitar el proceso de enseñanza/aprendizaje de dicha función comunicativa, así como tener en cuenta el orden de adquisición de los mecanismos de modalidad externa y su secuenciación.

Además, la atención a los tres criterios básicos a los que responde la selección del modo en español debe hacerse de forma gradual y ordenada, sin olvidar que están conectados. Así pues, primeramente, debería hacerse una aproximación semántica que 
aporte un significado general del modo. En segundo lugar, una sintáctica que muestre las condiciones estructurales, aunque al mismo tiempo están sometidas a un criterio semántico, por las que aparecen determinadas realizaciones. Finalmente, una pragmática que establezca diferencias de carácter semántico para realizaciones en las que la selección modal aparece determinada por la intención del hablante.

\section{Referencias bibliográficas}

Alarcos Llorach, E. (1970) [1984], Estudios de gramática funcional del español, Madrid: Gredos.

Bañón Hernández, A. M. (1999), "Apuntes para el análisis sintáctico-semántico de la locución a lo mejor en español hablado", en J. A. Samper Padilla (ed.), Actas del XI Congreso Internacional de la Asociación de Lingüística y Filología de la América Latina, Las Palmas de Gran Canaria (1996), Vol. 1, Universidad de Las Palmas de Gran Canaria: Servicio de Publicaciones, 137-146.

Briz, A. (1995), "La atenuación en la conversación coloquial. Una categoría pragmática”, en L. Cortés (ed.), El español coloquial. Actas del I Simposio sobre análisis del discurso oral, Almería: Servicio de Publicaciones, 103-122.

Carbonero Cano, P. (1997), "Significado Modal y Significante modal”, en Introducción Teórica a la Pragmática Lingüistica, Sevilla: Editorial Kronos, 66-71.

Castañeda Castro, A. (2004a), "Implicaturas generalizadas de cantidad en el rendimiento de algunas formas y oposiciones del sistema verbal español", Language Design: Journal of Theoretical and Experimental Linguistics, $\mathrm{n}^{\circ} 5,79-103$.

Castañeda Castro, A. (2004b), "Una visión cognitiva del sistema temporal y modal del verbo español”, ELUA, Estudios de Lingüística, Anexo 2, 55-71.

Langacker, R. (1991), Foundations of Cognitive Grammar, Vol. II, Stanford: University Press.

Matte Bon, F. (1992), Gramática comunicativa del español, Vol. I y II, Barcelona: Difusión.

Real Espinosa, J.M. (2005), “Tiempo y aspecto verbal: marco teórico para la clase de E/LE”, Marco ELE, Revista de Didáctica, [en 
línea] <http://marcoele.com/descargas/1/real_espinosa- tiempo-aspecto.pdf $>$ [fecha de consulta: 03.03.2018].

Ruiz Campillo (1998), La enseñanza significativa del sistema verbal: un modelo operativo. Universidad de Granada (tesis doctoral), Biblioteca RedELE, MEC, nº 1, 2004. 